\title{
Classificação baseada na técnica fuzzy integrado dados espectrais e de sensor radar
}

Anna Carolina Cavalheiro - Curso de Pós-Graduação em Ciências Geodésicas - CPCG, Setor de Ciências da Terra, Universidade Federal do Paraná - UFPR - Curitiba - Pr, annacarolina@ufpr.br

Diuliana Leandro - Curso de Pós-Graduação em Ciências Geodésicas - CPCG, Setor de Ciências da Terra, Universidade Federal do Paraná - UFPR - Curitiba - Pr, diuliana@ufpr.br

Jorge Centeno - Curso de Pós-Graduação em Ciências Geodésicas - CPCG, Setor de Ciências da Terra, Universidade Federal do Paraná - UFPR - Curitiba - Pr, centeno@ufpr.br

\section{RESUMO}

A integração de imagens sensores como ALOS/Palsar e CBERS tem potencial de aplicação no monitoramento e conservação ambiental, no mapeamento geológico e em pesquisas relacionadas a recursos minerais entre outros. A classificação dessas imagens por meio da lógica fuzzy permite inferir funções de pertinência que associa um pixel a determinada classe baseada num grau de pertinência. Neste trabalho pretende-se estudar a técnica de classificação baseada na lógica fuzzy integrando dados espectrais e de sensor radar, pois, dessa forma é considerada a natureza heterogênea e imprecisa dos dados utilizados.

\section{Classification technique based on fuzzy integrated spectral data and radar sensor}

\begin{abstract}
The integration of image sensors such as ALOS / PALSAR and CBERS has potential application in monitoring and environmental conservation, in geological mapping and in research related to mineral resources among others. The classification of these images using of fuzzy logic membership functions to inference associating the pixels to a class based on a degree of relevance. This paper aims to study the classification technique based on fuzzy logic using integrating spectral data and radar sensor, considered heterogeneous nature and imprecise of the data.
\end{abstract}




\section{INTRODUÇÃO}

As técnicas tradicionais de Sensoriamento Remoto utilizam somente informação espectral para classificação de imagens, mas através de técnicas como: lógica fuzzy, árvores de decisão, redes neurais, algoritmos genéticos podem ser incluídas informações de outras fontes de dados que podem facilitar procedimentos como a segmentação e a classificação, gerando melhorias na interpretação e conseqüente extração de informações.

Devido a difícil implementação de algoritmos capazes de realizar integração de dados de múltiplas fontes na classificação de imagens instiga a busca por técnicas de fácil aplicação. Neste trabalho pretende-se estudar a técnica de classificação baseada na lógica fuzzy integrando dados espectrais e de sensor radar.

Muitos estudos têm utilizado a integração desses dados através da fusão de imagens Radar e multiespectral, buscando sintetizar uma nova imagem com melhor resolução espectral e espacial que a original. Atualmente os trabalhos relacionados a esse tipo de fusão se concentram em técnicas no domínio espectral, como, por exemplo, o método de Componente Principais e Multiplicativo. Segundo Junior et AL (2005) o método de Componentes principais é o que vem apresentando melhores resultados, pois com ele se obtêm melhor nitidez e realce das feições de interesse.

A integração dos dados desses sensores tem apresentado grande potencial de aplicação no monitoramento e conservação ambiental, no mapeamento geológico e em pesquisas minerais. Por exemplo, segundo Filho et al (2006) o uso combinado do SAR e do sensor hiper-espectral das aeronaves de sensoriamento remoto do projeto Sivam-Censipam permitirá a análise simultânea de dados de alta resolução espacial e espectral , com o objetivo de estudar anomalias geo- botânicas associadas a campos de gás na bacia do rio Solimões. Esse projeto permitirá a implantação de um sistema de vigilância dos rios e da zona costeira amazônica, onde o sensoriamento remoto pode ser considerado a única fonte de dados que fornece informações em múltiplas escalas de tempo e espaço.

Estudos relacionados a classificação de imagens utilizando lógica fuzzy vem sendo realizados por Melgani et al. (2000); Bárdossy e Samaniego (2002); Cazes (2005). Estes trabalhos apresentam como ponto comum o método de classificação supervisionada baseada em regras fuzzy utilizando as características espectrais das imagens para a geração das funções de pertinência.

Neste trabalho não será realizada a fusão desses dados de Radar e da imagem multiespectral e sim se utilizará os dados de ambas as fontes aplicando lógica fuzzy para a integração desses dados, essa abordagem segundo Jensen (1996) considera a natureza heterogênea e imprecisa do mundo real e, pode ser utilizada juntamente com algoritmos de classificação supervisionada e não supervisionada. E Noguchi (2004) afirma que a teoria fuzzy também chamada teoria nebulosa tem se destacado cada vez mais na área de processamento de imagens, pois proporciona uma ferramenta satisfatória na representação de incertezas que surgem em segmentação ou classificação, sendo possível através dela modelar a atividade de percepção dos seres humanos.

\section{Classificação por lógica fuzzy}

Segundo Chuvieco (1990, p. 325), para a maior parte dos usuários de sensoriamento remoto, a classificação é a fase culminante do tratamento digital de imagens. Esta importância se dá porque os valores numéricos adquiridos pelo sensor normalmente não são auto-interpretáveis. Mas se convertidos em classes que representem as informações 
contidas no terreno, transformam-se num produto cartográfico multifinalitário.

Nos métodos clássicos de classificação considerava-se como principio básico que um pixel pertence plenamente a uma classe, ou seja, aplicava-se a lógica ocidental, binária, isto é, uma declaração é falsa ou verdadeira, o pixel pertencia ou não pertencia a determinada classe. No entanto, essa lógica não representa o que realmente ocorre, pois os dados são naturalmente misturados e imprecisos. Melgani (2000) assume que um pixel não é uma unidade na imagem indecomponível, que um pixel pode pertencer a várias classes. Essa abordagem assume que é possível obter mais informações sobre o pixel ao contrário dos métodos duros classificação que são pobres em informação extração.

Dentro desse contexto, apresenta-se a lógica fuzzy, a qual abrange um conjunto de conceitos e metodologias que permitem processar informações subjetivas (Zadeh, 1965). Essa lógica adota a teoria dos Conjuntos Zadeh, a qual considera que um conjunto não apresenta seus limites bem definidos, ao contrário da teoria clássica dos conjuntos em que a transição entre conjuntos ocorre abruptamente. Nos conjutos Zadeh, esta transição ocorre de forma gradual, não existe uma fronteiras nítida definida entre eles, e um elemento pode pertencer com certo grau a um conjunto, podendo este grau variar entre zero e um (Kronbauer et al; 2005).

\subsection{Métodos de Inferência Fuzzy}

O processo de inferência fuzzy é o processo de elaboração do mapeamento de uma dada entrada a uma saída usando lógica fuzzy, envolvendo todas as fases de elaboração do sistema. Dentre os sistemas de interferencia existentes dois apresentam maior destaque, Mamdani e Sugeno. Estes diferem na forma como determinam os resultados dos dados analisados.
O método Mamdani foi proposto por Ebrahim Mamdani em 1975, é o método mais usualmente utilizado com a metodologia fuzzy. O método combina os graus de pertinência referentes a cada um dos valores de entrada, através do operador mínimo e agrega as regras através do operador máximo. Este método de inferência de permite converter em conjunto fuzzy até mesmo dados de entrada em formato crisp. Este fato é que sugere a defuzzificação do conjunto fuzzy de saída para se obter um valor crisp que o represente.

De uma forma geral, o modelo fuzzy Sugeno, consiste da descrição de um sistema não linear como a combinação fuzzy de um certo número de modelos locais lineares e invariantes no tempo, sendo que estes modelos descrevem aproximadamente o comportamento deste sistema em diferentes pontos do espaço de dados. A modelagem fuzzy Sugeno também pode descrever exatamente certas classes de sistemas não-lineares.

\section{Satélite ALOS/PAlsar}

O satélite ALOS foi lançado em 24 de janeiro de 2006, pela Agência Espacial Japonesa (JAXA), possui 3 sensores a bordo: o radiômetro PRISM (Panchromatic Remote-Sensing Instrument for Stereo Mapping) capaz de adquirir imagens tridimensionais da superfície terrestre, o radiômetro multiespectral AVNIR-2 (Advanced Visible and Near Infrared Radiometer-2) e o sensor de microondas PALSAR (Phased Array type L-band Synthetic Aperture Radar).

O sensor PALSAR a bordo do ALOS opera em banda L, com resolução de 10 a 100 $\mathrm{m}$, capaz de realizar observações noturnas e diurnas, independentemente das condições meteorológicas, bem como interferometria com uma segunda passagem na área de interesse.

O PALSAR pode operar num modo ScanSAR com uma faixa de imageamento 
de 250 a $300 \mathrm{Km}$, que é 3 a 5 vezes mais largo do que os instrumentos SAR convencionais. Possui um modo polarimétrico que é capaz de gerar imagens com polarizações $\mathrm{HH}, \mathrm{HV}, \mathrm{VV}$ e VH. Por ser um sensor de radar, é capaz de gerar imagens mesmo sobre regiões cobertas por nuvens e à noite.

\section{CBERS}

O Programa CBERS - China-Brasil Earth Resources Satellite agrega a capacidade técnica e os recursos financeiros do Brasil e da China com a finalidade de estabelecer um sistema completo de sensoriamento remoto competitivo e compatível com as necessidades internacionais atuais.

O lançamento do primeiro satélite CBERS ocorreu 1999, utilizando-se o foguete chinês Longa Marcha 4B. A orbita do sistema CBERS é de $778 \mathrm{~km}$ com uma inclinação de 98,5 graus. O referido satélite possui os seguintes sistemas imageadores: CCD - High Resolution (Câmera de alta resolução), IR-MSS - Infrared Multispectral Scanner (Câmera de varredura no infravermelho) e WFI - Wide Field Imager (Imageador de Largo Campo de Visada).

A câmera CCD fornece imagens de uma faixa de $113 \mathrm{~km}$ de largura, com uma resolução de $20 \mathrm{~m}$. Esta câmera tem capacidade de orientar seu campo de visada dentro de \pm 32 graus, possibilitando a obtenção de imagens estereoscópicas de uma certa região. Além disso, qualquer fenômeno detectado pelo WFI pode ser focalizado pela Câmera CCD, para estudos mais detalhados, através de seu campo de visada, no máximo a cada três dias. Esta câmera opera em 5 faixas espectrais incluindo uma faixa pancromática de 0,51 a $0,73 \mu \mathrm{m}$. As duas faixas espectrais do WFI são também empregadas na câmera CCD para permitir a combinação dos dados obtidos pelas duas câmeras. São necessários 26 dias para uma cobertura completa da Terra.

\section{MÉTODOLOGIA}

\section{1 Área de Estudo}

A área de estudo abrange a cidade e a região metropolitana de Curitiba, no estado do Paraná. A cena é mesclada por uma região de vegetação e área urbana composta por um conjunto diversificado de materiais, tais como: concreto, vegetação rasteira, árvores, solo, edificações.

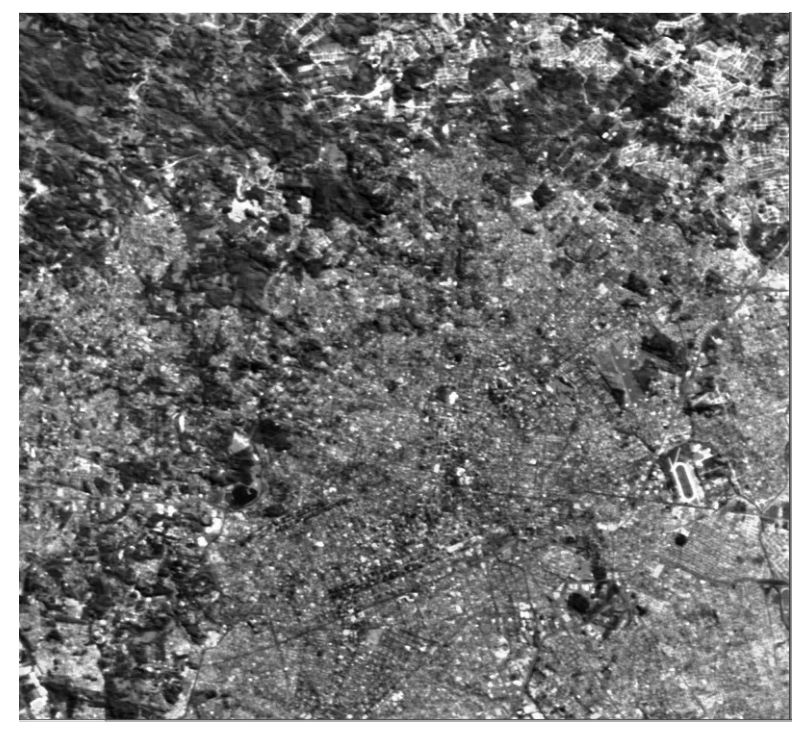

Figura 1. Área de estudo.

\subsection{Materiais e Procedimentos}

Para a realização do estudo desenvolveu-se uma rotina em MatLab para aplicação da logica fuzzy na classicação de dados de múltiplas fontes. Foram utilizadas uma imagem de Radar ALOS com resolução de 12,5 metros e uma imagem multiespectral CBERS com resolução de 20 metros, a partir da qual determinou-se o índice de vegetação normalizado.

Através do toolbox Editor FIS, se determinou as regras de interferência fuzzy gerais, criou-se: as entradas, as regras de pertinência e as saídas. Como entradas se utilizou a imagem NDVI e a Radar e baseadas nessas entradas foram determinados os parâmetros para as regras de pertinência. 
O parâmetros índice de vegetação normalizado (NDVI), foi utilizado para salientar as áreas de vegetação, através de operações aritméticas entre as bandas do vermelho na região do visível e uma segunda do infravermelho próximo. Pois segundo Centeno (2004), o quociente NDVI (Fórmula 1) pode ser utilizado para discriminar vegetação de outras coberturas como o solo.

$$
\begin{aligned}
& \mathrm{NDVI}_{\mathrm{i}}=\frac{\mathrm{P}_{\mathrm{i}}-\mathrm{V}_{\mathrm{i}}}{\mathrm{P}_{\mathrm{i}}+\mathrm{V}_{\mathrm{i}}} * \mathrm{a}+\mathrm{b} \\
& \text { Sendo: } \\
& \mathrm{P}_{\mathrm{i}} \quad \text { banda no infravermelho próximo; } \\
& \mathrm{V}_{\mathrm{i}} \quad \text { banda do vermelho; } \\
& \mathrm{a}, \mathrm{b} \text { fatores para escalar resultado. }
\end{aligned}
$$

O segundo parâmetro utilizado foi a intensidade da imagem de radar, o qual permite a descriminação principalmente de áreas urbanas de outras feições. Nesta imagem os as áreas urbanas apresentaram variação de intensidade de 2000 a 40000, as áreas de vegetação variaram entre 1300 e 9000 .

Estes parâmetros foram compostos na estrutura de indicadores quantitativos, que submetidos ao sistema de iInferência fuzzy, possibilitando a classificação da cena em áreas de vegetação, água e urbano.

\section{DESENVOLVIMENTO, ánalise e resul- tados}

O primeiro passo no desenvolvimento da rotina para a classificação integrando os dados Radar a imagem multiespectral foi a geração do NDVI. Como se pode observar na Figura 2, a geração do NDVI permitiu salientar as áreas de vegetação, devido ao grande contraste das bandas do vermelho e infravermelho.

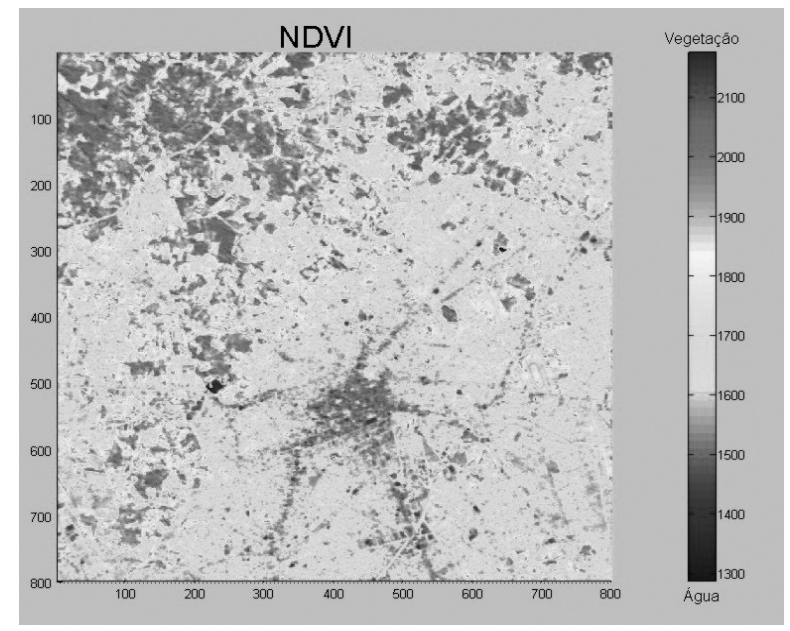

Figura 2. NDVI.

Posteriormente se definiu as regras de interferência e o como ocorrerá o fluxo de informações no sistema fuzzy de Mamdani, optando-se por duas entradas NDVI e Radar (Figura 3).

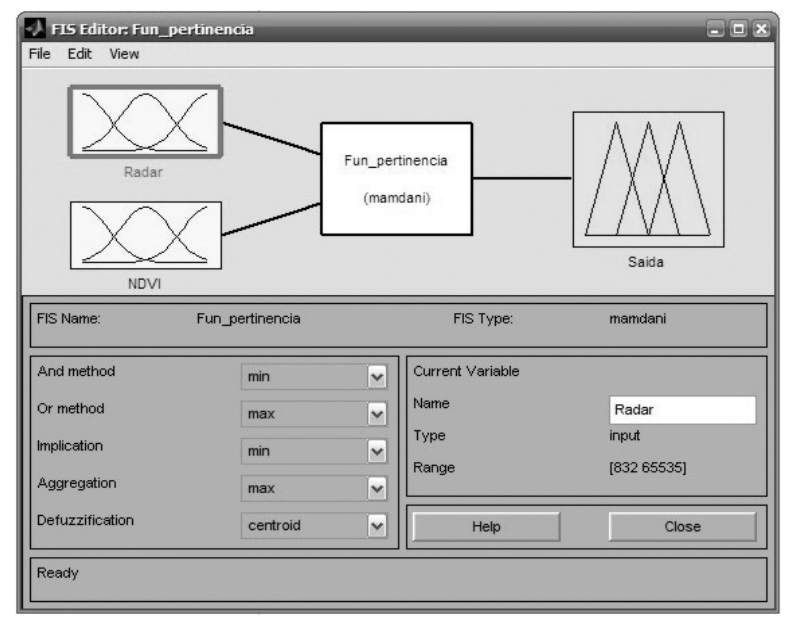

Figura 3. Descrição do processo de inferência fuzzy e do fluxo de informações.

Nesta etapa se iniciou o módulo de fuzzificação, no qual as variáveis de entrada foram modeladas matematicamente por meio de conjuntos fuzzy, ou seja, cada variável de entrada foi atribuído a um termo lingüístico.

A imagem Radar foi associada a três termos lingüísticos: escura, média, clara através de funções de pertinência triangulares que assume valores dentro do intervalo [0,1] (Figura 4). 


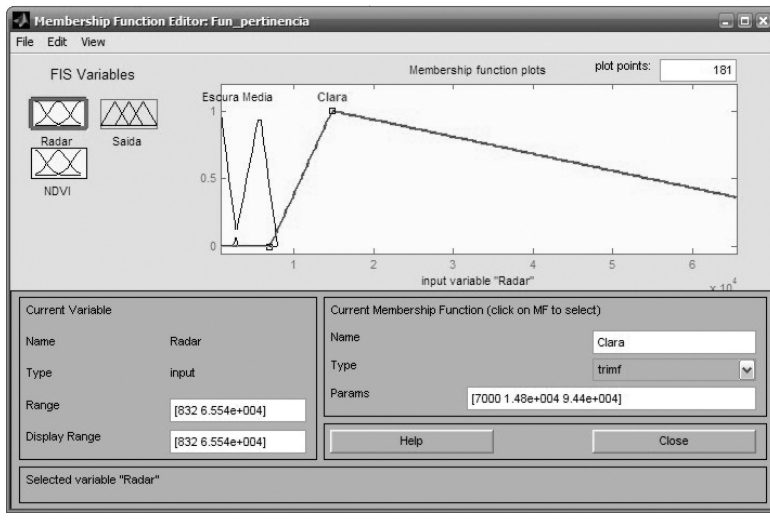

Figura 4. Funções de pertinência triangulares para a imagem Radar.

Para a imagem NDVI foram escolhidas funções de pertinência triangulares associadas aos termos lingüísticos: baixo, média, alta (Figura 5).

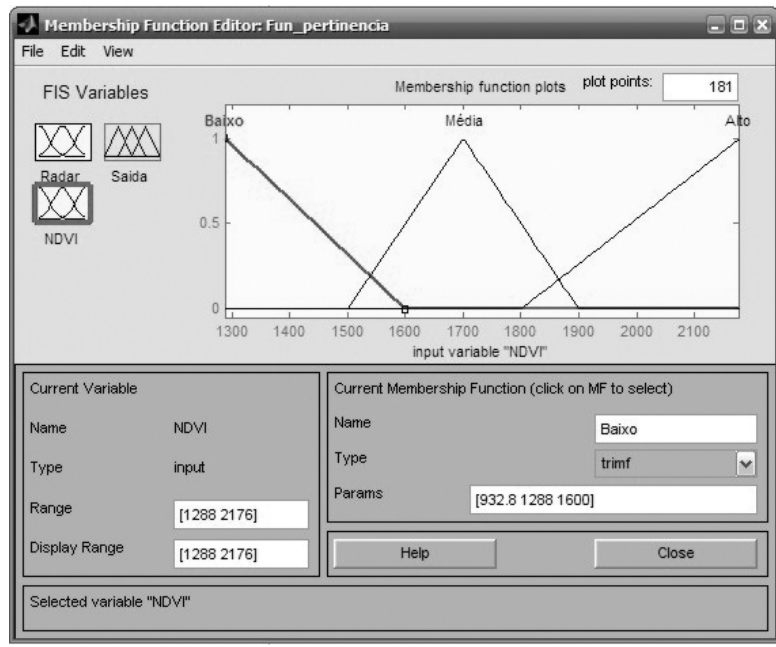

Figura 5. Variáveis lingüísticas associadas ao NDVI.

Da mesma maneira que para o NDVI e o Radar se criou funções de pertinência triangulares para a saída de dados associando as seguintes variáveis lingüísticas: vegetação, urbano e água (Figura 6).

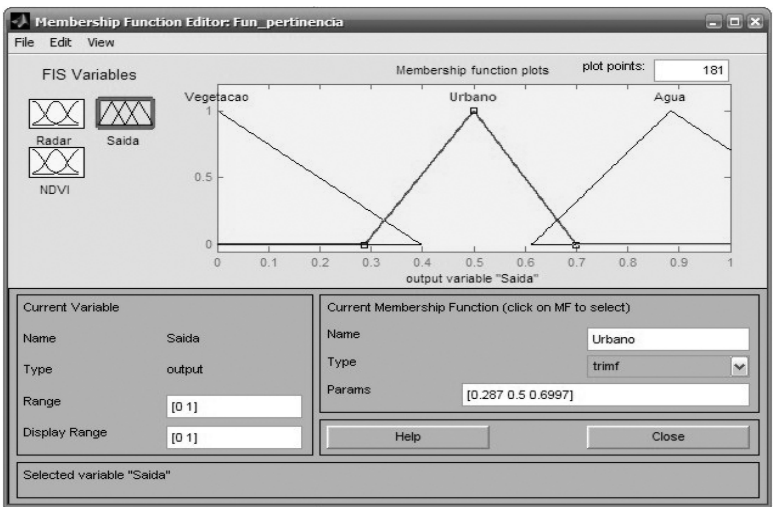

Figura 6. Variáveis lingüísticas associadas a saída de dados.

A etapa seguinte foi a definição da base de regras para o sistema. Neste módulo foram escritas as regras que associam as variáveis de entradas e suas classificações lingüísticas a variável de saída, como a integração de diferentes fontes foram associadas para a geração de uma nova informação (Figura 7).

A Tabela 1 apresenta as 8 combinações entre os conjuntos Fuzzy referentes a classificação dos dados em vegetação, urbano e água.

Tabela 1. Regras para o sistema 1.

\begin{tabular}{|l|}
\hline $\begin{array}{l}\text { 1. If (Radar is Escura) and (NDVI is Alto) then } \\
\text { (Saida is Vegetacao) }\end{array}$ \\
\hline $\begin{array}{l}\text { 2. If (Radar is Escura) and (NDVI is Baixo) then } \\
\text { (Saida is Agua) }\end{array}$ \\
\hline $\begin{array}{l}\text { 3. If (Radar is Media) and (NDVI is Alto) then (Saida } \\
\text { is Vegetacao) }\end{array}$ \\
\hline $\begin{array}{l}\text { 4. If (Radar is Media) and (NDVI is Média) then } \\
\text { (Saida is Urbano) }\end{array}$ \\
$\begin{array}{l}\text { 5. If (Radar is Clara) and (NDVI is Média) then } \\
\text { (Saida is Urbano) }\end{array}$ \\
\hline $\begin{array}{l}\text { 6. If (Radar is Clara) and (NDVI is Baixo) then } \\
\text { (Saida is Urbano) }\end{array}$ \\
\hline $\begin{array}{l}\text { 7. If (Radar is Escura) and (NDVI is Média) then } \\
\text { (Saida is Vegetacao) }\end{array}$ \\
$\begin{array}{l}\text { 8. If (Radar is Media) and (NDVI is Baixo) then } \\
\text { (Saida is Urbano) }\end{array}$ \\
\hline
\end{tabular}




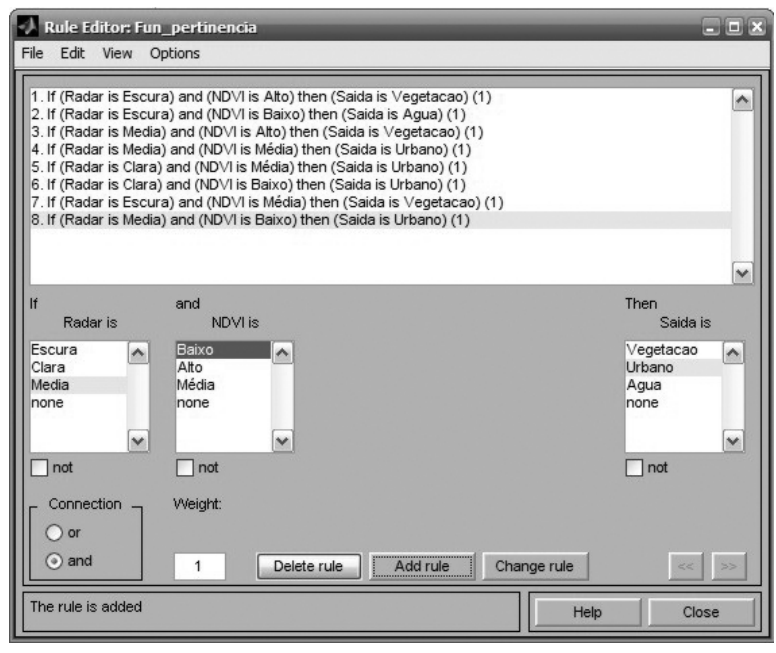

Figura 7. Base de regras para a classificação.

De acordo como as oito combinações estabelecidas na base de regras, o sistema traduz o estado da variável de saída fuzzy para um valor numérico. Por exemplo, se a entrada de Radar possuir o valor 33200 e o valor no NDVI for 1730, o sistema acionará a base de regras e trará como retorno o valor de saída 0,495 que representa a classe urbana (Figura 8).

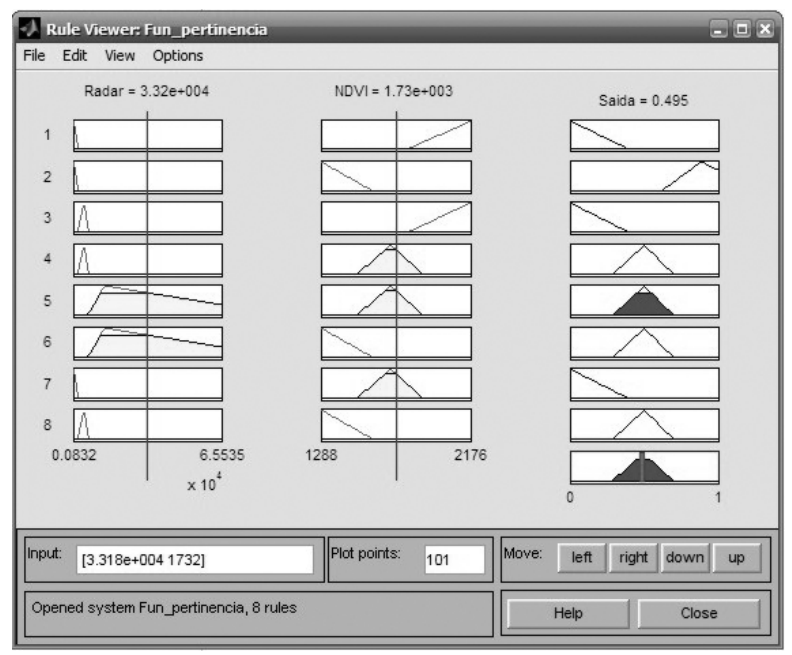

Figura 8. Exemplo das combinações na base de regras.

Através do módulo Surface Viewer é possível a visualização 3D dos dados de entrada da imagem Radar e do NDVI, e a saída de dados. Esse mapeamento permiti quantifi- car os dados de saída por classe. Na Figura 9 observa-se que a maior parte dos dados de saída foram classificados como urbano, seguido dos dados de vegetação e em menor quantidade os dados relacionados a classe água.

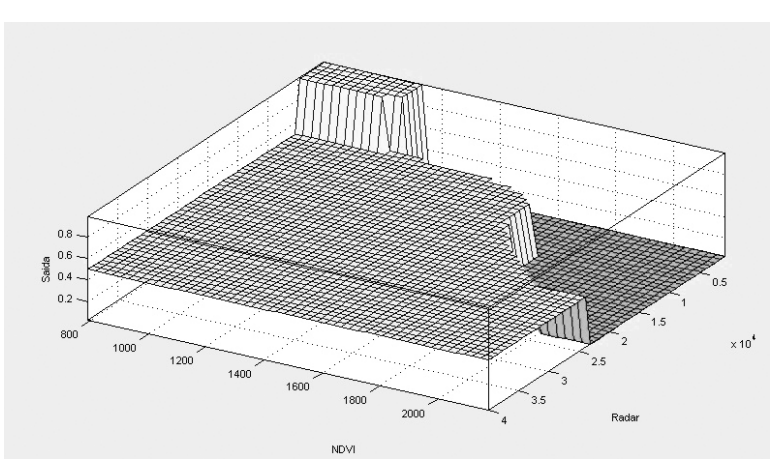

Figura 9. Mapeamento 3D dos dados de saida.

Com os vetor dos dados de saída foi possível gerar uma imagem classificada. $\mathrm{Na}$ Figura 10, visualiza-se o resultado da classificação utilizando a integração de dados de múltiplas fontes através da lógica fuzzy.

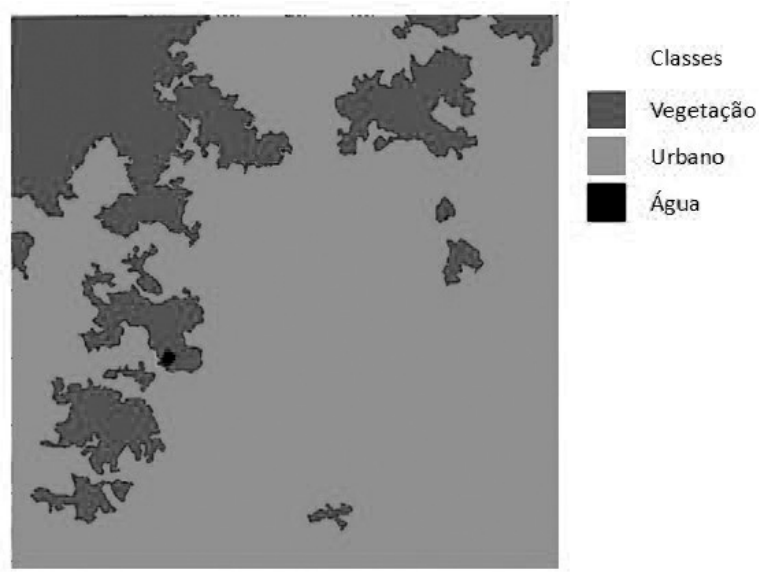

Figura 10. Resultado da classificação.

\section{Considerações Finais}

O presente artigo relatou as etapas para a criação de uma rotina em MatLab para a classificação de dados de múltiplas fontes utilizando um sistema baseado em regras fuzzy.

Essa metodologia gerou bons resultados, além de vantagens, como a possibilidade no caso de erros nos resultados interpretados 
pelas regras ser possível efetuar correções nas mesmas, buscando um resultado melhor. A inserção de novas regras ao sistema também é simplificada. Porém a determinação dessas regras é dependente da experiência do especialista.

\section{REFERÊNCIAS}

AMENDOLA, M.; SOUZA, A.L.; BARROS, L.C. Manual do uso da teoria dos conjuntos fuzzy no matlab 6.5. 2005. 46 p. Disponível em: <www.ime.unicamp.br/ laeciocb/ MANUAL_2005.pdf/>. Acesso em: 27 junho 2009.

AMENDOLA, M. ; SOUZA, Anderson Luiz de . Manual do uso da Teoria dos Conjuntos Fuzzy no Matlab 6.1. In: III Ciclo de Palestras: Modelagem Matemática e Simulação Numérica para Suporte à Decisão em Sistemas da Engenharia Agrícola, 2004, Campinas. Oficina - Uso da Teoria Fuzzy no Matlab para sistemas pré definidos em zootecnia de precisão, 2004.

BÁrdossy, A.; SAMANIEGO, L. Fuzzy Rule-Based Classification of Remotely Sensed Imagery. IEEE Transactions on Geoscience and Remote Sensing, v. 40, n. 2, p. 362-374, fev. 2002.

CAZES, T. B. Interpretação Baseada em Conhecimento de Imagens de Sensores Remotos de Alta Resolução. Dissertação (mestrado em Engenharia Elétrica), 89P., PUC-Rio, 2005.

CENTENO, J. A. da S. Sensoriamento Remoto e Processamento de Imagens Digitais. Editora Curso de Pós Graduação em Ciências Geodésicas, Universidade Federal do Paraná, Curitiba, 2004.
FILHO, P., PARADELLA, W., SOUZA Jr., C., VALERIANO, D. M. \& MIRANDA, F. P. Sensoriamento Remoto e recursos naturais da Amazônia. Ciência e Cultura, 2006.

JÚNIOR , A. G; PEREIRA, A. A. C.; DALL'IGNA, L. G. Fusão de imagens CBERS2-SAR/SIPAM para identificação de campo de pouso na região amazônica. XII Simpósio Brasileiro de Sensoriamento Remoto - SBSR . Goiânia - GO, 2005.

MATHWORKS. Fuzzy Logic Toolbox ${ }^{\text {TM }}$ User's Guide. 2009. Disponível em < http:// www.mathworks.com/access/helpdesk/help/ pdf_doc/fuzzy/fuzzy.pdf>. Acessado em: 27 de junho de 2009.

MELGANI, F.; AL HASHEMY, B.A.R.; TAHA, S.M.R. An Explicit Fuzzy Supervised Classification Method for Multispectral Remote Sensing Images. IEEE Transactions on Geoscience and Remote Sensing, v. 38, n. 1, p. 287-295, jan. 2000.

MOREIRA, M. L. O. ; COSTA H.F. ; MEDEIROS, L. C. Integração de Dados Multifontes Para Mapeamentos Temáticos. In: XII Simpósio Brasileiro de Sensoriamento Remoto, 2005, Goiânia. Anais XII Simpósio Brasileiro de Sensoriamento Remoto, 2005.

NOGUCHI, M. T. G. Um sistema baseado em regras fuzzy para classificação supervisionada de imagens multiespectrais de alta resolução. 2004. Dissertação (Mestrado em Informática) - Universidade Federal do Paraná, 2004.

KRONBAUER, A. H. ; FERREIRA, L. C. ; NUNES JUNIOR, R. . Mineração de Dados Utilizando Lógica Fuzzy. Ciente-fico.com, v. II, p. 87-91, 2005. 
MATHWORKS. Fuzzy Logic Toolbox ${ }^{\text {TM }}$

User's Guide. 2009. Disponível em < http://

www.mathworks.com/access/helpdesk/help/

pdf_doc/fuzzy/fuzzy.pdf> . Acessado em: 27

de junho de 2009. 